\title{
Validación y comparación de los puntajes TIMI y GRACE en pacientes con síndrome coronario agudo sin elevación del segmento ST
}

\author{
Juan M. Sénior ${ }^{\mathrm{a}, \mathrm{b}, *}$, Andrés Fernández ${ }^{\mathrm{a}, \mathrm{c}}$, Arturo Rodríguez ${ }^{\mathrm{a}, \mathrm{c}}$, Edison Muñoz $^{\mathrm{a}, \mathrm{c}}$, \\ James Díaz ${ }^{\mathrm{a}, \mathrm{b}}$, Jairo Gándara ${ }^{\mathrm{a}, \mathrm{b}}$, Marta C. Cardona ${ }^{\mathrm{a}, \mathrm{d}}$, Gilma N. Hernández ${ }^{\mathrm{a}, \mathrm{e}}$ \\ y Fabián Jaimes ${ }^{a, e}$
}

a Grupo para la Investigación de Enfermedades Cardiovasculares, Sección Cardiología, Departamento de Medicina Interna, Universidad de Antioquia, Medellín, Colombia

b Posgrado de Cardiología Clínica y Cardiología Intervencionista, Universidad de Antioquia, Medellín, Colombia

c Hospital Universitario San Vicente Fundación, Medellín, Colombia

d Unidad de Dolor Torácico, Hospital Universitario San Vicente Fundación, Medellín, Colombia

e Epidemiología Clínica y Estadística, Universidad de Antioquia, Medellín, Colombia

Recibido el 25 de septiembre de 2015; aceptado el 18 de abril de 2016

Disponible en Internet el 11 de julio de 2016

\section{PALABRAS CLAVE}

Síndrome coronario agudo;

Validación;

Pronóstico

\begin{abstract}
Resumen
Introducción: La validación y comparación de los modelos de predicción clínica es recomendable puesto que pueden tener diferente desempeño en las poblaciones de acuerdo con características étnicas, socio-demográficas, genéticas, culturales o idiosincráticas.

Metodología: Estudio de validación y comparación de dos escalas de pronóstico en una cohorte prospectiva de pacientes mayores de 18 años de edad con diagnóstico de síndrome coronario agudo sin elevación del segmento ST.

Resultados: Se incluyeron 507 pacientes en el análisis. El promedio de edad fue de $65 \pm 11$ años, el $54,4 \%$ tenía más de 65 años, el $55,6 \%$ era de sexo masculino, el $43,8 \%$ tenía al menos tres factores de riesgo coronario y el tipo de síndrome coronario fue angina inestable en un $52,3 \%$ e infarto de miocardio sin elevación del segmento ST en un 47,7\%. La distribución de mortalidad hospitalaria $(n=21,4,1 \%)$ por cualquier causa, contrasta entre los diversos grupos de riesgo, con diferencias significativas para ambos puntajes (TIMI $p=0,00001$; GRACE $p=0,0016$ ); al igual que para el GRACE extrahospitalario $(p=0,00001)$. La calibración de los modelos fue adecuada (Hosmer Lemeshow > 0,05). La discriminación del desenlace aislado de muerte fue buena para ambas escalas en el escenario intrahospitalario (AUC-ROC TIMI 0,75 vs. GRACE 0,79, $p=0,37$ ), con diferencias significativas a 30 días (AUC-ROC TIMI 0,71 vs. GRACE 0,85, $p=0,0049$ ) y a 6 meses (AUC-ROC TIMI 0,75 vs. GRACE 0,84, $p=0,0194$ ) a favor de la escala GRACE.
\end{abstract}

\footnotetext{
* Autor para correspondencia.

Correo electrónico: mmbt@une.net.co (J.M. Sénior).
} 
Discusión: Ambas escalas muestran desempeño similar en el ambiente intrahospitalario. El modelo GRACE tiene mejor capacidad predictiva al mes y a los 6 meses.

(๖) 2016 Publicado por Elsevier España, S.L.U. en nombre de Sociedad Colombiana de Cardiología y Cirugía Cardiovascular. Este es un artículo Open Access bajo la licencia CC BY-NC-ND (http:// creativecommons.org/licenses/by-nc-nd/4.0/).

\section{KEYWORDS}

Acute coronary syndrome; Validation; Prognosis
Validation and comparison of TIMI and GRACE scores in patients with acute coronary syndrome with no ST-segment elevation

\begin{abstract}
Introduction: It is advisable to validate and compare clinical prediction models because they can perform differently according to the ethnic, socio-demographic, genetic, cultural and idiosyncratic characteristics of populations.

Methodology: A validation and comparison study of prognostic scales in a prospective cohort of patients over the age of 18 with a diagnosis of acute coronary syndrome with no ST-segment elevation.

Results: five hundred and seven patients were included in the study. The mean age was $65 \pm 11$, $54.4 \%$ were aged over $65,55.6 \%$ were males, $43.8 \%$ had at least three coronary risk factors, and the type of coronary syndrome was unstable angina in $52.3 \%$ and myocardial infarction with no ST-segment elevation in $47.7 \%$. Between the different risk groups there is a contrast in the distribution of hospital mortality $(n=21,41 \%)$ due to any cause, with significant differences for both scores (TIMI $p=0.00001$; GRACE $p=0.0016)$; likewise for the outpatient GRACE $(p=0.00001)$. The models were appropriately calibrated (Hosmer Lemeshow $>0.05$ ). Discrimination of isolated outcome of death was good for both scales in an inpatient setting (AUC-ROC TIMI 0.75 vs GRACE 0.79, $p=0.37$ ), with significant differences at 30 days (AUC-ROC TIMI 0.71 vs GRACE 0.85, $p=0.0049$ ) and at 6 months (AUC-ROC TIMI 0.75 vs GRACE $0.84, p=0.0194$ ) in favour of the GRACE scale.

Discussion: Both scales perform similarly in an inpatient setting. The GRACE model has greater predictive capability at one month and at 6 months.

(c) 2016 Published by Elsevier España, S.L.U. on behalf of Sociedad Colombiana de Cardiología y Cirugía Cardiovascular. This is an open access article under the CC BY-NC-ND license (http: / / creativecommons.org/licenses/by-nc-nd/4.0/).
\end{abstract}

\section{Introducción}

La predicción en medicina ha sido un tema central, especialmente en el área cardiovascular. Un modelo predictivo que estratifique el riesgo de incidencia de un evento, establece grupos que le permiten al clínico no solo conocer la probabilidad de desenlaces, sino escoger las alternativas diagnósticas y terapéuticas óptimas y la intensidad del tratamiento de acuerdo con el balance riesgo/beneficio de cada opción ${ }^{1}$.

En este sentido, se han utilizado diversos puntajes para determinar y cuantificar el pronóstico en pacientes con síndrome coronario agudo ${ }^{2} \mathrm{y}$, aunque emplean variables similares, se distinguen en algunas que se consideran importantes. Otra diferencia en el desarrollo de algunas de estas escalas es que se han obtenido en ensayos clínicos con asignación aleatoria, que están limitados por los criterios de inclusión utilizados, en contraposición a los puntajes construidos con base en registros internacionales, multicéntricos, con mayor representación de la población del llamado "mundo real" $3-9$.

Los puntajes más conocidos son el GRACE (The Global Registry of Acute Coronary Events) y el TIMI (Thrombolysis
In Myocardial Infarction). El primero ${ }^{8-10}$ fue desarrollado con base en un registro internacional, multicéntrico, con participación de 123 hospitales de 14 países, en pacientes con síndrome coronario agudo (SCA) con y sin elevación del segmento ST para estratificar el riesgo de infarto y muerte a un mes y a seis meses. El segundo ${ }^{6}$ se diseñó con base en dos estudios de intervención en SCA sin elevación del ST: el TIMI 11 ${ }^{11}$ y el ESSENCE ${ }^{12}$. Los dos modelos anteriores se usan de manera rutinaria en los servicios de urgencias para determinar el subgrupo de pacientes con infarto agudo del miocardio sin elevación del segmento ST/angina inestable (IAMNST/AI) de más alto riesgo, a quienes se les debe realizar estratificación invasiva ${ }^{13}$. No todas las cohortes a través del mundo han obtenido resultados iguales con la aplicación de estos puntajes ${ }^{14}$. En Iberoamérica se han realizado estudios con resultados variables ${ }^{15-17}$. En Colombia se han hecho dos aproximaciones a la validación de los puntajes TIMI y GRACE con resultados contradictorios ${ }^{18,19}$.

El objetivo de este estudio fue determinar si el puntaje de riesgo GRACE predice con mayor exactitud que el TIMI, el riesgo de muerte como desenlace aislado y el combinado de muerte o reinfarto no fatal en el escenario intrahospitalario, al mes y a los seis meses. 


\section{Materiales y métodos}

\section{Diseño del estudio}

Estudio de validación de dos puntajes de pronóstico con una cohorte prospectiva de pacientes mayores de 18 años de edad con diagnóstico de síndrome coronario agudo atendidos en la unidad de cuidados coronarios de dos instituciones de alta complejidad de la ciudad de Medellín desde enero de 2013 hasta septiembre de 2014.

\section{Criterios de inclusión}

Pacientes con SCA sin elevación del segmento ST: angina inestable o infarto agudo de miocardio sin elevación del segmento ST, que se presentaron con angina típica clasificada como inestable por las siguientes características: angina en reposo de más de 20 minutos de duración en las 48 horas previas al ingreso, de reciente comienzo (últimos 60 días) o in crescendo (que haya cambiado un estado funcional de la clasificación canadiense de angina y sea mínimo estado III), más uno de los siguientes:

- Cambios electrocardiográficos: depresión del segmento ST $\geq 0,1 \mathrm{mV}$ de más $80 \mathrm{~ms}$ de duración en dos derivaciones contiguas o elevación transitoria del segmento $\mathrm{ST} \geq$ $0,1 \mathrm{mV}$ o inversión de la onda $\mathrm{T}$ profunda $\geq 0,1 \mathrm{mV}$.

- Marcadores enzimáticos de necrosis positivos: troponina I por encima del percentil 99 (valores de referencia de la prueba en ambas instituciones).

- En pacientes con angina sin cambios eléctricos y enzimas negativas, se necesita para la inclusión al menos un criterio de alto riesgo: edad $\geq 60$ años, infarto de miocardio previo, revascularización percutánea o quirúrgica previa, enfermedad arterial coronaria previa documentada mayor al $50 \%$ en al menos un vaso, enfermedad cerebrovascular isquémica previa o ataque isquémico transitorio, estenosis carotídea mayor al 50\%, enfermedad arterial periférica, diabetes mellitus, disfunción renal crónica definida como depuración de creatinina menor a $60 \mathrm{ml}$ por minuto por $1,73 \mathrm{~m}^{2}$ de superficie corporal.

\section{Criterios de exclusión}

Se excluyeron pacientes que rehusaran participar en el estudio o asistir a las citas de seguimiento, pacientes con expectativa de vida menor a 6 meses, pacientes con SCA con elevación persistente del segmento ST, pacientes con angina de Prinzmetal o portadores de marcapasos.

\section{Tamaño de la muestra}

La aproximación convencional al cálculo del tamaño de la muestra para los modelos predictivos, define un valor entre 5 y 20 desenlaces por cada variable independiente ${ }^{20,21}$. Sin embargo, para la indicación específica de validación de modelos predictivos no existe evidencia que sustente la consideración anterior, aunque algunos autores sugieren al menos un número similar de desenlaces y no desenlaces ${ }^{22}$. Dado que el objetivo general del estudio parte de la hipótesis de una mayor exactitud de una escala versus la otra, se tomaron como referencia los valores originales de áreas bajo la curva ROC (AUC-ROC de los dos modelos $\mathrm{TIMI}=0,63$ y GRACE $=0,85)^{6,8}$. Para probar la hipótesis de la diferencia entre las dos anteriores AUC-ROC, con error alfa de 0,05 y beta de 0,2 , y con base en la fórmula de Hanley-Mc Neil y Obuchowsky ${ }^{23,24}$, se necesitan alrededor de 119 individuos para el cálculo de cada una de las dos AUC-ROC para una población total de al menos 238 pacientes.

\section{Análisis estadístico}

La distribución de las variables continuas se describe con medidas de tendencia central y de dispersión, y para las variables discretas se hace con frecuencias y porcentajes. Para la validación de los modelos predictivos se utilizaron las mismas estrategias de los estudios originales. Para el puntaje TIMI se ajustó un modelo de regresión logística con las variables dicotómicas descritas originalmente y para el GRACE un modelo de regresión logística con la introducción de las variables originales con sus respectivos puntajes. En ambos modelos se usó el desenlace compuesto de muerte y reinfarto no fatal, al igual que el de muerte en forma aislada, intrahospitalaria, al mes y a los 6 meses.

Para la validación de los dos modelos se analizó su capacidad discriminativa y su calibración. Para la discriminación, respecto a los desenlaces descritos previamente, se realizó un área bajo la curva de las características operativas del receptor (AUC-ROC), considerando clínicamente importante un valor $\geq 0,7$. Para la calibración se hizo una prueba de bondad de ajuste de Hosmer Lemeshow con 10 deciles de riesgo ( 8 grados de libertad), en la cual se esperan valores de $p$ altos $(>0,05)$ que indiquen que no hay diferencias significativas entre las frecuencias de eventos observadas y las predichas por el modelo para cada grupo de riesgo. Para la comparación de las curvas AUC-ROC de los dos modelos se utilizó una prueba de igualdad de las áreas bajo la curva con el algoritmo de DeLong, DeLong y Pearson, con estadístico $\mathrm{Chi}^{2}$ y $\mathrm{p}<0,05^{25}$.

\section{Ética}

El protocolo de investigación fue aprobado por los comités de ética de las instituciones participantes y no se requirió consentimiento informado, dado que se clasifica como estudio de "riesgo mínimo"', según el numeral b del Artículo 11, de la resolución 008430 de 1993 del Ministerio de Salud de la República de Colombia.

\section{Resultados}

Se analizaron 507 pacientes, con promedio de edad de $65 \pm$ 11 años; el 54,4\% $(n=276)$ tenía más de 65 años, el $55,6 \%$ $(n=282)$ era de sexo masculino, el $43,8 \%(n=222)$ tenía al menos tres factores de riesgo coronario y el tipo de síndrome coronario fue angina inestable en un $52,3 \%(n=265)$ e infarto de miocardio sin elevación del segmento ST en un $47,7 \%(n=242)$, con distribuciones similares a las del registro GRACE (tabla 1). 
Tabla 1 Características de la población de estudio (pacientes con SCA sin elevación del segmento ST, Medellín 2013-2014)

\begin{tabular}{|c|c|c|}
\hline Características & Cohorte validación (507) & GRACE (32.037) \\
\hline \multicolumn{3}{|l|}{ Demográficas } \\
\hline Edad (años, rango) & $65(28-93)$ & $66,6(56-76,4)$ \\
\hline Sexo masculino\% (n) & $55,6(282)$ & $67(21464)$ \\
\hline Peso (kg, rango) & $68,6(35-115)$ & $78(68-89)$ \\
\hline Talla (cm, rango) & $163(135-186)$ & $170(162-175)$ \\
\hline IMC $\left(\mathrm{kg} / \mathrm{m}^{2}\right.$, rango $)$ & $25,8(14,9-40,4)$ & $27(24-30)$ \\
\hline \multicolumn{3}{|l|}{ Historia médica } \\
\hline Angina $(\%, \mathrm{n})$ & $65,3(331)$ & 44 \\
\hline Cirugía de puentes coronarios $(\%, n)$ & $6,1(6,1)$ & 13 \\
\hline Falla cardiaca $(\%, n)$ & $12,4(63)$ & 10 \\
\hline Diabetes $(\%, n)$ & $30(152)$ & 26 \\
\hline Dislipidemia $(\%, n)$ & $53,5(271)$ & 51 \\
\hline Hipertensión $(\%, n)$ & $76,9(390)$ & 64 \\
\hline Enfermedad coronaria $(\%, \mathrm{n})$ & $33,9(172)$ & 30 \\
\hline Intervención coronaria percutánea $(\%, n)$ & $17,9(91)$ & 19 \\
\hline Enfermedad arterial periférica $(\%, n)$ & $7,5(38)$ & 9 \\
\hline Falla renal $(\%, \mathrm{n})$ & $11,8(60)$ & 7,6 \\
\hline Tabaquismo $(\%, \mathrm{n})$ & $51,3(260)$ & 57 \\
\hline Enfermedad cerebrovascular $(\%, n)$ & 3,7 (19) & 8,5 \\
\hline \multicolumn{3}{|l|}{ Ingreso } \\
\hline Pulso (lpm) & $79(37-200)$ & $76(65-90)$ \\
\hline Presión arterial sistólica (mm Hg) & $138(66-250)$ & $140(120-160)$ \\
\hline Presión arterial diastólica (mm Hg) & $78,6(40-178)$ & $80(70-90)$ \\
\hline Killip । (\%) & 87,6 & $85 \%$ \\
\hline Killip ॥ (\%) & 5,9 & $11 \%$ \\
\hline Killip III (\%) & 3,7 & $3,6 \%$ \\
\hline Killip Iv (\%) & 2,8 & $0,8 \%$ \\
\hline Paro cardiaco (\%) & 3,4 & $1,9 \%$ \\
\hline Biomarcadores positivos (\%) & 49,3 & $52 \%$ \\
\hline Creatinina inicial (mg/dL) & $1,6(0,4-90)$ & $1,02(0,90-1,25)$ \\
\hline Cambios electrocardiográficos (\%) & 41,8 & \\
\hline Uso previo de ASA (\%) & 46,4 & 40 \\
\hline Tipo de síndrome coronario agudo: angina inestable/infarto sin ST (\%) & $52,3 / 47,7$ & \\
\hline
\end{tabular}

* Tomado de: Fox et al. ${ }^{26}$. ASA: ácido acetil salicílico.

\section{Manejo intrahospitalario}

Los siguientes son los resultados de las ayudas diagnósticas solicitadas al ingreso:

- Troponinas: $100 \%$ de la medición fue convencional (no ultrasensible), con niveles promedio de 6,43 $\pm 24,5 \mathrm{ng} / \mathrm{l}$.

- Hemoglobina inicial: $13,8 \pm 2 \mathrm{~g} / \mathrm{dl}$.

- Hematocrito: 40,9 $\pm 5,6 \%$.

- Glicemia: $139 \pm 96 \mathrm{mg} / \mathrm{dl}$ (383 pacientes).

- Hemoglobina glicosilada: 7,3 \pm 3,9\% (358 pacientes).

- Creatinina: 1,6 $\pm 5,14 \mathrm{mg} / \mathrm{dl}$.

- Depuración de creatinina promedio: $68 \pm 30 \mathrm{ml} / \mathrm{m}$.

- Niveles de péptido natriurético cerebral: $681 \pm$ $1840 \mathrm{pg} / \mathrm{dl}$ (98 pacientes).

- Fracción de eyección: $54 \pm 15 \%$ (rango 10-84).

El 6,5\% (33 pacientes) tuvo complicaciones relacionadas con el síndrome coronario agudo, $4,5 \%(n=23)$ de tipo eléctrico y $2 \%$ (10) mecánico. De los pacientes ingresados a tan solo el 2,2\% (11 pacientes) no se les realizó angiografía coronaria y se les dio manejo médico. De los 496 pacientes estratificados en forma invasiva, el 69,8\% (346 pacientes) tenía lesión en al menos una arteria coronaria mayor del $50 \%$ y de estos $34(9,6 \%)$ recibieron solo manejo médico, el $78 \%$ intervención coronaria percutánea y el $12,4 \%$ requirió cirugía de puentes coronarios. El 6,3\% $(n=30)$ tuvo alguna complicación relacionada con la angiografía, de las cuales el 2,8\% (14 pacientes) correspondió a hematoma del sitio de punción, el 1,6\% (8 pacientes) a nefrotoxicidad, el $0,4 \%$ ( 2 pacientes) a seudoaneurisma, el $0,4 \%$ ( 2 pacientes) a ruptura arterial radial y el 0,2\% (1 paciente) a disección de arteria radial.

En cuanto a medicamentos prescritos durante la hospitalización y al alta el $97,9 \%$ recibió un inhibidor del receptor P2Y12, el 94,5\% clopidogrel (479), el 0,2\% ( $n=1)$ prasugrel y el 3,2\% ( $n=16)$ ticagrelor; el 97,4\% ( $n=494)$ ASA, el $92,3 \%$ terapia antiagregante dual, el 16\% $(n=81)$ inhibidor de glicoproteína IIb/IIla (tirofibán), el 92,1\% (n=467) un inhibidor de la enzima convertidora de angiotensina/bloqueador de receptor II de angiotensina, el 92,9\% $(n=471)$ un betabloqueador (metoprolol o carvedilol), el 33,5\% ( $n=170)$ nitratos, el $96,4 \%(n=489)$ estatinas, el 47,1\% $(n=239)$ diuréticos, el $30,2 \%(n=153)$ un calcioantagonista y el $24,5 \%(n=124)$ un 
antialdosterónico. Para la anticoagulación se utilizó: enoxaparina $93,3 \%(n=473)$, heparina intravenosa $30,6 \%(n=155)$ y el $4,3 \%(n=22)$ fondaparinux. Otros medicamentos menos utilizados fueron: digoxina 2,2\% $(n=11)$, inotrópicos $25,4 \%$ (129) y vasopresores $7,5 \%(n=38)$.

\section{Desenlaces}

Durante la hospitalización, de 507 pacientes ingresados se presentaron 5 reinfartos (1\%), 8 intervenciones percutáneas urgentes $(n=1,6 \%)$ y dos casos de enfermedad cerebrovascu$\operatorname{lar}(n=0,4 \%)$. Fallecieron 21 pacientes $(4,1 \%), 3,7 \%(n=19)$ de causa cardiovascular y $0,4 \%(n=2)$ de causa no cardiovascular. Se observó algún tipo de sangrado en el 4,7\% $(n=24)$ de los casos, de los cuales $2 \%(n=10)$ fueron sangrado mayor. En el primer mes, de 500 pacientes en seguimiento se presentaron 17 casos de reinfarto, uno de enfermedad cerebrovascular y 6 de sangrado; la frecuencia de muertes acumulada fue de 27 pacientes $(5,4 \%), 4,4 \%$ y $1 \%$ de causa cardiovascular y no cardiovascular, respectivamente. A los seis meses, de 482 pacientes que completaron el seguimiento se presentaron 34 casos de reinfarto, 3 de enfermedad cerebrovascular y 7 de sangrado; la frecuencia de muertes acumulada fue de 37 pacientes $(7,6 \%), 6,6 \%$ y $1 \%$ de causa cardiovascular y no cardiovascular, respectivamente.

\section{Estratificación de riesgo por las escalas TIMI y GRACE}

Los 507 pacientes de la cohorte se clasificaron según la escala TIMI en riesgo bajo 2,17\% (11 pacientes), intermedio $77,91 \%$ (395 pacientes) y alto $19,92 \%$ (101 pacientes); la escala GRACE clasificó los pacientes intrahospitalarios en riesgo bajo 0,2\% ( 1 paciente), intermedio 65,29\% (331 pacientes) y alto $34,52 \%$ (175 pacientes), en tanto que los extra-hospitalarios en riesgo bajo 0,2\% (1 paciente), intermedio $65,48 \%$ (332 pacientes) y alto $34,32 \%$ (174 pacientes). De acuerdo con la estratificación por TIMI se revascularizó al 36\% (100\% percutáneo) de los pacientes de riesgo bajo, al $60 \%$ de riesgo intermedio (85\% percutáneo y $15 \%$ quirúrgico) y al $76 \%$ de riesgo alto (90\% percutáneo y $10 \%$ quirúrgico); por la escala GRACE se revascularizó al $0 \%$ de riesgo bajo, al $62 \%$ de riesgo intermedio ( $84 \%$ percutáneo y $16 \%$ quirúrgico) y al $65 \%$ de riesgo alto ( $90 \%$ percutáneo y $10 \%$ quirúrgico).

La distribución de mortalidad hospitalaria por cualquier causa difiere entre los diversos grupos de riesgo, con diferencias significativas tanto para el TIMI (chi ${ }^{2}$ homogeneidad 24,26 y $\mathrm{p}=0,0000,2 \mathrm{gl}$; $\mathrm{chi}^{2}$ de tendencia 22,5 y $\mathrm{p}=0,0000,1$ $\mathrm{gl}$ ), como para el GRACE (chi ${ }^{2}$ homogeneidad 10 y $\mathrm{p}=0,0067$, $2 \mathrm{gl}$; $\mathrm{chi}^{2}$ de tendencia 9,97 y $\mathrm{p}=0,0016,1 \mathrm{gl}$ ); al igual que para el GRACE extrahospitalario (chi ${ }^{2}$ homogeneidad 30,39 y $\mathrm{p}=0,0000,2 \mathrm{gl} ; \mathrm{chi}^{2}$ de tendencia 30,21 y $\mathrm{p}=0,0000,1 \mathrm{gl}$ (fig. 1).

\section{Calibración y discriminación de los puntajes}

\section{Desenlace mortalidad}

La calibración de los modelos fue adecuada en la población de estudio de acuerdo con el estadístico de HosmerLemeshow (tabla 2). La discriminación del desenlace aislado

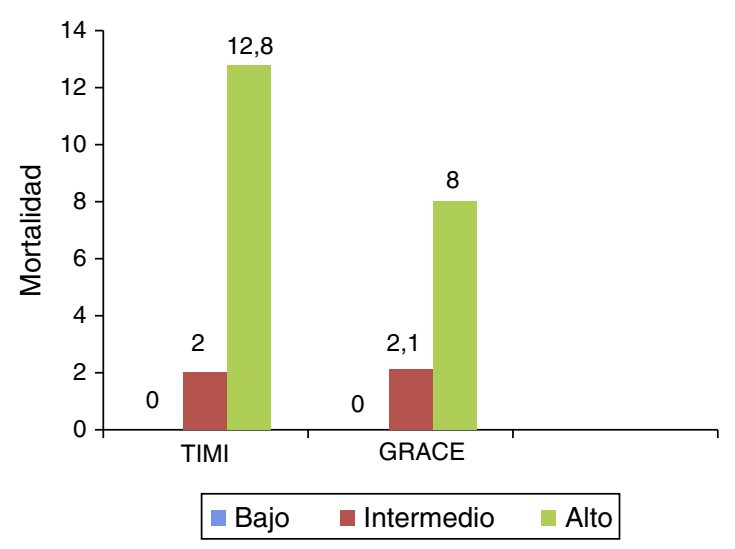

Figura 1 Mortalidad hospitalaria por cualquier causa de acuerdo con las escalas de estratificación de riesgo TIMI y GRACE.

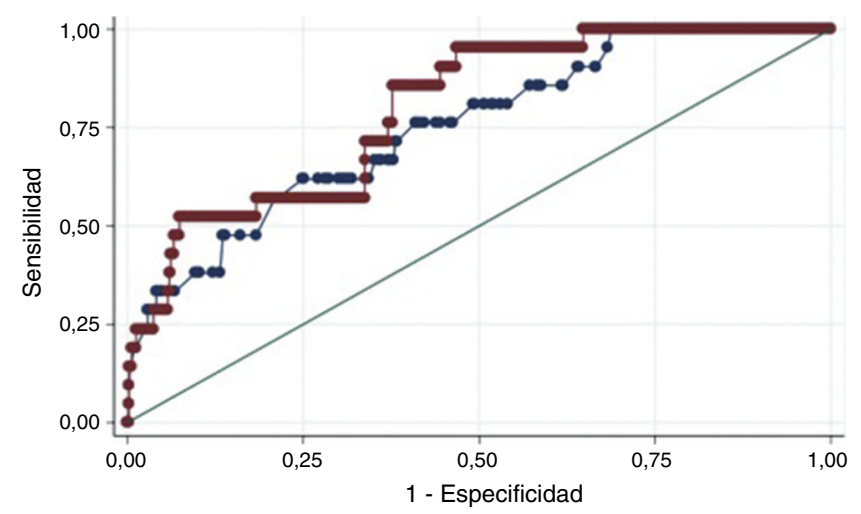

Figura 2 Comparación curva ROC TIMI y GRACE para desenlace de mortalidad intrahospitalario.

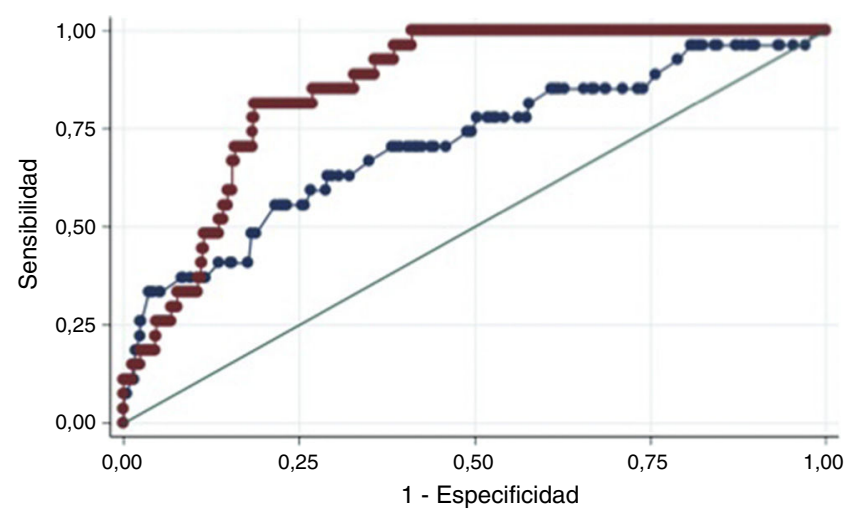

Figura 3 Comparación curva ROC TIMI y GRACE para desenlace de mortalidad al mes.

de muerte fue buena para ambos puntajes en el escenario intrahospitalario, al mes y a 6 meses. Sin embargo, hay diferencias significativas de los modelos al mes y a 6 meses, a favor de la escala GRACE que conserva un AUC-ROC mayor de 0,8 (figs. 2-4).

\section{Desenlace mortalidad o reinfarto}

La discriminación del desenlace combinado de muerte o reinfarto fue adecuada para ambas escalas en el escenario intrahospitalario, con AUC-ROC de 0,73 (IC 95\% 0,64-0,82) 
Tabla 2 Calibración y capacidad discriminativa de los modelos de predicción TIMI y GRACE intrahospitalario, al mes y a los 6 meses para mortalidad

\begin{tabular}{lllll}
\hline & HL,p & AUC-ROC & IC 95\% & $\mathrm{p}^{*}$ \\
\hline TIMI intrahospitalario & 0,75 & 0,7513 & $0,64-0,85$, \\
GRACE intrahospitalario & 0,97 & 0,7972 & $0,70-0,88$ \\
TIMI 1 mes & 0,86 & 0,71 & $0,59-0,82$ & 0,37 \\
GRACE 1 mes & 1 & 0,85 & $0,80-0,90$ & 0,0049 \\
TIMI 6 meses & 0,43 & 0,75 & $0,65-0,82$ & 0,0194 \\
GRACE 6 meses & 1 & 0,84 & $0,78-0,89$ \\
\hline
\end{tabular}

HL,p: valor de p correspondiente a un estadístico de Hosmer-Lemeshow con 8 grados de libertad; AUC-ROC: área bajo la curva ROC.

Prueba de igualdad de las áreas bajo la curva con el algoritmo de DeLong, DeLong y Pearson.

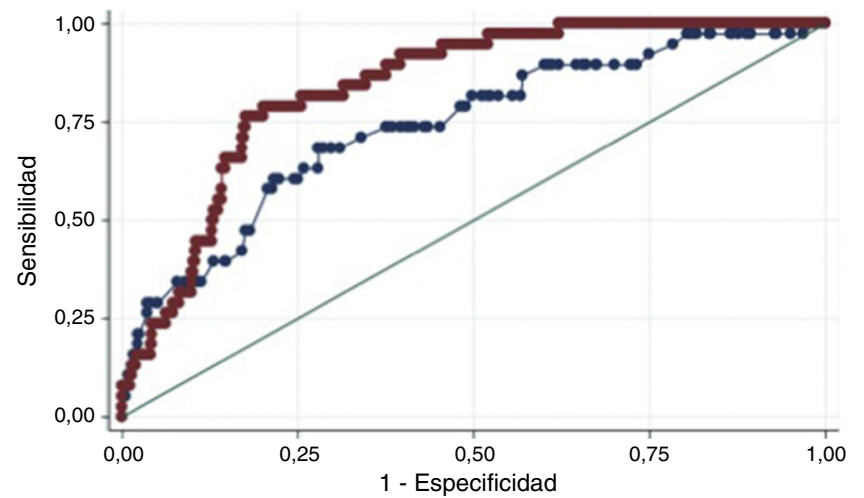

Figura 4 Comparación curva ROC TIMI y GRACE para desenlace de mortalidad a seis meses.

para la escala TIMI y de 0,76 (IC 95\% 0,67-0,85) para la escala GRACE $\left(\mathrm{Chi}^{2} \mathrm{0,34}, \mathrm{p}=0,55\right)$, con comportamiento similar al observado con el desenlace de muerte aislado al mes y a seis meses.

\section{Discusión}

Los resultados de este estudio confirman el valor predictivo de dos puntajes utilizados rutinariamente en la evaluación médica en el servicio de urgencias de los pacientes con síndrome coronario agudo sin elevación del segmento ST, con hallazgos a favor del puntaje GRACE al mes y a los 6 meses en comparación con el TIMI. Las variables utilizadas en ambos puntajes son de fácil obtención en el contacto médico inicial, lo que permite estratificar con rapidez el riesgo de muerte y por ende la utilización de estrategia intervencionista temprana; sin embargo, el puntaje TIMI posee la ventaja de su fácil aplicación en servicios de alto volumen de pacientes.

La validación de este tipo de puntajes es necesaria puesto que en la práctica muchos de ellos no funcionan igual en poblaciones diferentes para las que fueron desarrollados, por diferencias étnicas, socio-demográficas, genéticas, culturales o idiosincráticas entre otras ${ }^{27}$. Adicionalmente, el espectro de tratamiento para el síndrome coronario agudo ha cambiado en forma importante desde el desarrollo y la publicación de los puntajes, si bien el modelo predictivo GRACE ha sido actualizado recientemente ${ }^{26}$.

En los estudios que sirvieron de base para el desarrollo del puntaje TIMI, los estudios TIMI 11B y ESSENCE, fue revascularizado el $11,1 \%$ y el $32,2 \%$ de los pacientes, respectivamente, y en el GRACE alrededor del 34\% de los pacientes recibieron esta misma intervención. En nuestra cohorte se estratificaron en forma invasiva la mayoría de los pacientes, de los cuales $69,8 \%$ tenían compromiso de al menos un vaso de más, $50 \%$ en al menos un vaso, el $78 \%$ fue revascularizado en forma percutánea y el $12,4 \%$ quirúrgica. La revascularización miocárdica tiene mayor efecto en la reducción del desenlace de infarto de miocardio no fatal que en el de mortalidad, ya sea como desenlace aislado o combinado en el seguimiento (reducción del riesgo absoluto del 2-3,8\% en riesgo bajo e intermedio vs. $11,1 \%$ en riesgo alto ${ }^{28}$. Otro aspecto paradójico reportado en la literatura es la subutilización de la estrategia invasiva en pacientes estratificados en riesgo alto o intermedio ${ }^{29}$, en contravía con el efecto benéfico demostrado en este grupo de pacientes y a pesar del aumento en el número de procedimientos percutáneos a través del tiempo. En nuestra cohorte se revascularizó el $63 \%$ de los pacientes, con tendencia a mayor intervención de acuerdo con el nivel de riesgo, la mayoría en forma percutánea, de los cuales un $65 \%$ se clasificó en riesgo alto según la escala GRACE y un $76 \%$ en riesgo alto según la TIMI, en contraposición al $0 \%$ y $36 \%$ de los pacientes en riesgo bajo por GRACE y TIMI, respectivamente.

Diversos ensayos clínicos han demostrado el impacto de ciertos medicamentos en la evolución y el pronóstico de los pacientes con síndrome coronario agudo, cuya adherencia ha mejorado desde los primeros reportes del registro GRACE $^{30}$ hasta nuestros días, aunque persisten diferencias regionales $^{31}$. El patrón de tratamiento con medicamentos es similar al reportado en otros países respecto al uso de betabloqueador: 76\% Reino Unido (UK), 88,6\% Suecia (SW), $89,4 \%$ Estados Unidos (EU) y $92,9 \%$ en la cohorte (C); uso de antiagregantes plaquetarios 94,6\% UK, 94,5\% SW, 95,4\% EU y $97,9 \%$ C; terapia antiagregante dual: $76,2 \%$ UK, $68,1 \%$ SW, $66,4 \%$ EU y $92,3 \%$ C; estatinas: $91,5 \%$ UK, $81,1 \%$ SW $84,5 \%$ EU y $96,4 \%$ C; IECA/ARA ॥ 78,7\% UK 68,8\% SW, 64,5\% EU y $92,1 \%$ C. Sin embargo, conviene aclarar que los datos son extraídos de registros como el MINAP (UK), RIKS-HIA (SW) y ACTION (EU) y contrastarlos con datos de una cohorte seguida en dos unidades de manejo especializado podría no reflejar la situación real de la población colombiana ${ }^{31}$.

La estratificación del riesgo es discretamente diferente de otras series como la validación española ${ }^{17}$, en la cual se reporta riesgo bajo por GRACE en el 12,8\%, intermedio en el $26,7 \%$ y alto en el $60,5 \%$, en contraposición con la nuestra con $0,20 \%, 65,29 \%$ y $34,52 \%$ respectivamente, diferencias 
explicadas por el tipo de población incluida en hospitales de referencia. Se observó en la cohorte una tendencia clara al incremento en la frecuencia de desenlaces respecto al nivel de riesgo tanto en el puntaje TIMI como en el GRACE, similar a lo demostrado en su desarrollo y validación ${ }^{6}$. La exactitud en la predicción se determina por medio de dos medidas estadísticas complementarias y relacionadas pero diferentes: la discriminación y la calibración. En el análisis realizado se demostró adecuada calibración de los dos modelos (Hosmer-Lemeshow $>0,05$ ) en los distintos escenarios (intrahospitalario, un mes y seis meses) y para el desenlace aislado de mortalidad y el combinado de muerte o reinfarto, lo que implica que se ajusta a la población de estudio y las probabilidades observadas son cercanas a las esperadas, corroborando los datos iniciales encontrados en la cohorte retrospectiva ${ }^{19}$. Para la discriminación se utiliza el estadístico $\mathrm{C}$ o el área bajo la curva ROC, que es la proporción de pares de pacientes, uno sin desenlace y otro con desenlace, en el cual el paciente con el desenlace tiene la más alta probabilidad predicha del evento. Un modelo que discrimine con exactitud los pacientes $85 \%$ de las veces tendrá un estadístico $C$ de 0,85 ; predicciones aleatorias como al lanzar una moneda, tendrá un estadístico $C$ de 0,50 ; se considera que estadísticos $C$ de 0,6 o menos no tienen valor clínico, de 0,6-0,7 tienen valor limitado, de 0,7 a 0,8 tienen un valor modesto y mayores a 0,8 tienen un valor clínico importante ${ }^{32,33}$. En cuanto a la discriminación, los datos son similares a los publicados inicialmente para el TIMI en su artículo original $(0,74)$ con estadístico $C$ de 0,75 para mortalidad intrahospitalaria, pero con diferencias importantes para el desenlace combinado de muerte o reinfarto, con estadístico $C$ de 0,73 en la cohorte vs. 0,63 en el TIMI. Aunque el puntaje fue desarrollado para predicción a 14 días, ha sido validado a largo plazo ${ }^{34}$ y conserva un estadístico $C>$ de 0,7 a 30 días y 6 meses en nuestro estudio. Al puntaje GRACE se le ha reconocido su excelente capacidad de discriminación ${ }^{35}$, especialmente para el desenlace de mortalidad, corroborado por los hallazgos en la cohorte prospectiva actual, con estadístico $C>0,7$ intrahospitalario y $>0,8$ al mes y 6 meses.

La comparación de ambos puntajes demuestra desempeño similar en el ambiente intrahospitalario, con diferencias estadísticamente significativas al mes y a los 6 meses, explicado en parte por la forma en que se construyeron, con desenlaces en tiempos diferentes y con algunas variables también distintas para la predicción a mediano (6 meses) y largo plazo (1 y 3 años $)^{26}$.

\section{Limitaciones del estudio}

Una de las principales limitaciones del estudio, común en este tipo de investigaciones, es su realización en solo dos centros especializados, hecho que podría no reflejar el comportamiento en la población general y en otros centros de menor complejidad, sin disponibilidad de laboratorio de hemodinámica o adherencia a las recomendaciones de las guías de práctica clínica actuales. Aunque el tamaño de la muestra cumple con el cálculo realizado durante el diseño para la comparación de dos escalas, hubiese sido ideal poder construir un modelo de predicción con base en una mayor población de estudio. Pese a que la tasa de pérdidas fue muy baja, la predicción a 6 meses se relaciona no solo con las variables incluidas en los modelos, sino con otros factores de riesgo con datos longitudinales y medidas repetidas, que son difíciles de modelar, al igual que con factores relacionados con la adherencia al tratamiento y el acceso a la salud de la población sujeto de estudio. Por último, es importante señalar que aunque algunas de las variables del modelo GRACE tienen un comportamiento con tendencia lineal, no lo son completamente, en especial la frecuencia cardiaca, lo que implica dificultades en su ajuste en la regresión logística. Esta limitación, no obstante, está presente desde el desarrollo y la validación del modelo original y apenas se está considerando su corrección en una reciente investigación ${ }^{26}$.

\section{Responsabilidades éticas}

Protección de personas y animales. Los autores declaran que para esta investigación no se han realizado experimentos en seres humanos ni en animales.

Confidencialidad de los datos. Los autores declaran que en este artículo no aparecen datos de pacientes.

Derecho a la privacidad y consentimiento informado. Los autores declaran que en este artículo no aparecen datos de pacientes.

\section{Conflictos de interés y aspectos éticos}

La investigación fue financiada con recursos propios del Grupo para la Investigación de las Enfermedades Cardiovasculares, de la sección de Cardiología de la Universidad de Antioquia y no hay conflictos de interés por declarar.

Los aspectos éticos se ajustan a los principios éticos fundamentales y a las directrices de la Declaración de Helsinki y similares, emanados de la Asociación Médica Mundial, y sigue las pautas del Ministerio de la Protección Social de la República de Colombia según la resolución 8430 de 1993 por la cual se dictan las normas científicas, técnicas y administrativas para la investigación en salud.

\section{Bibliografía}

1. Steyeberg E. Applications of clinical predictors models. En: Steyerberg E, editor. Clinical predictors models. A practical approach to development, validation and updating. Statistics for biology abd health series. New York: Springer; 2009. p. 11-30.

2. Libby P, Theroux P. Pathophysiology of artery coronary disease. Circulation. 2005;111:3481-8.

3. Boersma E, Pieper KS, Steyerberg EW, Wilcox RG, Chang WC, Lee $\mathrm{KL}$, et al., for the PURSUIT Investigators. Predictors of outcome in patients with acute coronary syndromes without persistent ST-segment elevation: results from an international trial of 9461 patients. Circulation. 2000;101:2557-67.

4. Lagerqvist B, Diderholm E, Lindahl B, Husted S, Kontny F, Stahle $E$, et al. FRISC score for selection of patients for an early invasive treatment strategy in unstable coronary artery disease. Heart. 2005;91:1047-52.

5. Backus BE, Six AJ, Kelder JC. Chest pain in the emergency room; a multicenter validation of the HEART Score. Crit Pathways in Cardiol. 2010;9:164-9. 
6. Antman EM, Cohen M, Bernink PJ, McCabe CH, Horacek T, Papuchis G, et al. The TIMI Risk Score for Unstable Angina/Non-ST Elevation MI: a method for prognostication and therapeutic decision making. JAMA. 2000;284:835-42.

7. Morrow DA, Antman EM, Charlesworth A, Cairns R, Murphy SA, de Lemos JA, et al. TIMI risk score for ST-elevation myocardial infarction: a convenient, bedside, clinical score for risk assessment at presentation. Circulation. 2000;102:2031-7.

8. Granger C, Goldberg RJ, Dabbous O, Pieper KS, Eagle KA, Cannon $\mathrm{CP}$, et al. Predictors of hospital mortality in the global registry of acute coronary events. Arch Intern Med. 2003;163:2345-53.

9. Eagle KA, Lim MJ, Dabbous OH, Pieper KS, Goldberg RJ, Van de Werf F, et al. A validated prediction model for all forms of acute coronary syndrome: estimating the risk of 6month postdischarge death in an international registry. JAMA. 2004:291:2727-33.

10. Fox K, Dabbous O, Goldberg RJ, Pieper KS, Eagle KA, Van de Werf $\mathrm{F}$, et al. Prediction of risk of death and myocardial infarction in the six months after presentation with acute coronary syndrome: prospective multinational observational study (GRACE). BMJ. 2006;333:1091-4.

11. Antman E, McCabe C, Gurfinkel EP, Turpie AG, Bernink PJ, Salein $D$, et al. Enoxaparin prevents death and cardiac ischemic events in unstable angina/non $\mathrm{Q}$ wave myocardial infarction results of thrombolysis in myocardial infarction TIMI 11B trial. Circulation. 1999;100:1593-601.

12. Cohen M, Demers C, Gurfinkel EP, Turpie AG, Fromell GJ, Goodman S, et al. A comparison of low molecular weight heparin with unfractionated heparin for unstable coronary artery disease. N Engl J Med. 1997;337:447-52.

13. Sénior JM, Lugo LH, Acosta N, Saldarriaga C, Toro J, Diaz J, et al. Guía de práctica clínica para pacientes con diagnóstico de síndrome coronario agudo; atención inicial y revascularización. Rev Col Cardiol. 2013;20:45-85.

14. Filipiak K, Koltowski L, Grabowski M, Karpinski G, Glowczynska R, Huczek Z, et al. Prospective comparison of the 5 most popular risk scores in clinical use for unselected patients with acute coronary syndrome. Circ J. 2011;75:167-73.

15. Bagur RH, Urinovsky F, Contreras A, Estrada CD. Validación del score de riesgo TIMI para pacientes con síndrome coronario agudo sin elevación del segmento ST. MEDICINA (Buenos Aires). 2009;69:526-8.

16. Correia LC, Freitas R, Bittencourt AP, Souza AC, Almeida MC, Leal J, et al. Prognostic value of GRACE scores versus TIMI score in acute coronary syndromes. Arq Bras Cardiol. 2010;94(5):613-9.

17. Abu-Assi E, García-Acuña JM, Peña-Gil C, González-Juanatey JR. Validación en una cohorte contemporánea de pacientes con síndrome coronario agudo del score GRACE predictor de mortalidad a los 6 meses de seguimiento. Rev Esp Cardiol. 2010;63: 640-8.

18. Ocampo LA, Saldarriaga C, Gómez A, González C, González N. Exactitud pronóstica de las escalas GRACE y TIMI en pacientes llevados a intervencionismo percutáneo por síndrome coronario agudo sin elevación del ST. Rev Colomb Cardiol. 2013;20:130-5.

19. Aristizábal JC, Sénior JM, Fernández A, Rodríguez A, Acosta N. Validación de las escalas de riesgo TIMI y GRACE para el síndrome coronario agudo en una cohorte contemporánea de pacientes. Acta Med Colomb. 2014;39:336-43.
20. Vittinghoff E McCulloch. Relaxing the rule events per variable in logistic and cox regression. Am J Epidemiol. 2007;165:710-8.

21. Vergouwe Y, Steyeberg E, Eijkemans M, Habbena JDF. Substantial effective sample sizes were required for external validation studies of predictive logistic regression models. J Clin Epidemiol. 2005;58:475-83.

22. Moons KG, de Groot JA, Bouwmeester W, Vergouwe Y, Mallet S, Altman DG, et al. Critical appraisal and data extraction for systematic reviews of prediction modelling studies: The CHARMS Checklist. PLOS Medicine. 2014;11:e1001744.

23. Obuchowski N. Sample size in studies of test accuracy. Stat Methods Med Res. 1998;7:371-92.

24. Hanley J, McNeil B. A Method of comparing the areas under Receiver Operating characteristic curves derived from the same cases. Radiology. 1983;148:839-43.

25. De Long E, De Long D, Pearson DLC. Comparing the areas under two or more correlated receiver operating characteristic curves: a nonparametric approach. Biometrics. 1988;44:837-45.

26. Fox K, Fitzgerald G, Puymirat E, Huang W, Carruthers K, Simon T, et al. Should patients with acute coronary disease be stratified for management according to their risk? Derivation, external validation and outcomes using the updated GRACE risk score. BMJ Open. 2014;4:e004425.

27. Altman D, Royston P. What do we mean by validating a prognostic model? Statist Med. 2000;19:453-73.

28. Fox K, Clayton TC, Damman P, Pocock SJ, de Winter RJ, Tijsen $\mathrm{JG}$, et al. Long term outcome of a routine versus selective invasive strategy in patients with non-ST segment elevation acute coronary syndrome. J Am Coll Cardiol. 2010;55:2435-45.

29. Jedrzkiewicz S, Goodman S, Yan R, Weish RC, Korndern J, de Young JP, et al. Temporal trends in the use of invasive cardiac procedures for non-ST segment elevation acute coronary syndromes according to initial risk stratification. Can J Cardiol. 2009;25:e370-6.

30. Fox KAA, Goodman SG, Anderson FA, Granger CB, Moscucci M, Flather MD, et al. From guidelines to clinical practice: the impact of hospital and geographical characteristics on temporal trends in the management of acute coronary syndromes. The Global Registry of Acute Coronary Events (GRACE). Eur Heart J. 2003;24:1414-24.

31. McNamara RL, Chung SC, Jernberg T, Holmes D, Roe $M$, Timmis A, et al. International comparison of the management of patients with non ST segment elevation acute myocardial infarction in the United Kingdom, Sweden, and the United States: the MINAP/NICOR, SWEDEHEART/RISKS_HIA, and ACTION Registry-GWTG/NCDR registries. Int J Cardiol. 2014;175:240-7.

32. Harrell E Jr, Califf R, Pryor DB, Lee KL, Rosati RA. Evaluating the yield of medical tests. JAMA. 1982;247:2543-6.

33. Ohman E, Granger C, Harrington LA, Lee KL. Risk stratification and therapeutic decision making in acute coronary syndromes. JAMA. 2000;284:876-8.

34. Pollack C, Sites F, Shofer F, Sease K, Hollander JE. Application of the TIMI Risk score for unstable angina and non ST elevation acute coronary syndrome to an unselected emergency department chest pain population. Acad Emerg Med. 2006;13:13-8.

35. D'Ascenso F, Biondi-Zoccai G, Moretti C, Bollati M, Omedé P, Sciuto F, et al. TIMI, GRACE and alternative risk scores in acute coronary syndromes: a meta-analysis of 40 derivation studies on 216.552 patients and of 42 validation studies on 31.625 patients. Contemp Clin Trials. 2012;33:507-14. 